\title{
Reinforced Concrete Finite Element Analysis Incorporating Material Nonlinearity and Failure Criteria Aspects
}

\author{
Sri Tudjono ${ }^{1}$, Ay Lie Han², Lie Hendri Hariwijaya ${ }^{3}$ \\ ${ }^{1}$ Department of Civil Engineering, Diponegoro University, Prof. Soedarto SH, Semarang, Indonesia \\ ${ }^{2}$ Structural Laboratory, Diponegoro University, Prof. Soedarto SH, Semarang, Indonesia \\ ${ }^{3}$ Structural Laboratory, Diponegoro University, Prof. Soedarto SH, Semarang, Indonesia \\ 1tudjono@gmail.com; ²ayliehan@hccnet.nl; ${ }^{3}$ hendrihariwijaya@gmail.com
}

Keywords: Failure Criteria, Nonlinearity, Material Behavior.

\begin{abstract}
The behavior of concrete is highly nonlinear, even at very low loading levels. Steel, on the other hand, exhibits a relatively linear behavior up till yielding. The synergy between the two materials and their compatibility has long been the subject of research. While the failure criterion for steel is straight forward, concrete can be approached by various theories. The most prominent are the Kupfer-Hilsdorf-Rusch and the Möhr failure envelope. The behavior of material under bi-axial stresses subsequent to cracking can be assumed isotropic or orthotropic, resulting in a differentiation in the material constitutive matrix formulation. This work covers the finite element modeling of reinforced concrete elements, based on the two failure envelopes, while assessing the isotropic and orthotropic methodology. The Finite Element smeared crack approach is used to analyze stresses and the propagation of cracking pattern for the element. The resulting load - displacement curves are validated with identical laboratory tested specimens.
\end{abstract}

\section{Introduction}

The nonlinear behavior of concrete can be predicted and simulated by various methods. Among the widely used is the smeared crack approach that assumes an evenly distributed crack spreading, upon concrete failure. The crack direction is presumed perpendicular to the principal tensile stress. The cracked concrete however, is considered as a continuum, having a decreasing structural stiffness.

The advantage of the smeared crack method is its easiness in accommodating the reinforcing steel into the model. In finite element modeling, the contribution of steel reinforcement is taken into account by adding the steel stiffness modulus, resulting in an adjusted, integrated material stiffness.

At early loading stages, the material is elastic, having a constant modulus of elasticity. Nonlinearity will occur when stresses increase, resulting in a gradual decrease of the stiffness modulus. At further stages, the material behaves orthotropic, enabling a differentiation of stiffness modulus in the direction of the principal stresses. This orthotropic behavior is applied on the model which uses Möhr failure envelope. On the model which employs Kupfer-Hilsdorf-Rusch failure envelope, the material is assumed as isotropic, having an identical behavior in all directions. This is predominately true when stress combinations fall within this failure envelope. When exceeding the failure boundaries, adjustments to the material constitutive matrix are made.

\section{Failure Criteria}

\section{The Kupfer-Hilsdorf-Rusch Criteria}

Based on the principal stresses acting on a Gauss point, the Kupfer-Hilsdorf-Rusch [1] envelope distinguishes the failure mode into three criteria: bi-axial compression, bi-axial tension and compression-tension. The tension zone is characterized by cracking of concrete, when the major principal stress has reached the uni-axial tensile strength of the material. As for the bi-axial compression case, research has proven that a confinement is generated, increasing the ultimate compression strength to $20 \%$ of its uni-axial compression strength. The failure is distinguished by crushing of the concrete. The tension-compression region is the most intriguing, since the failure mode is defined by a combination of cracking in tension and crushing of concrete in compression. 


\section{The MöhrCriteria}

The Möhr criterion serves as failure envelope in the Möhr diagram. This criterion is represented by the envelope or curve that describes the critical state of stresses over a range of differential stresses [2]. Two exact critical states of stresses in concrete are acknowledged, these are the uni-axial tension and the uni-axial compression failure. Based on these two critical cases, the Möhr failure envelope is drawn. Although research has proven that the envelope is not exactly linear, in the analysis it is simplified linearly. Each state indicating the uni-axial tensile or compression failure is represented by an individual Möhr circle. If the tangent point at the Möhr circle for uni-axial compressive and tension failure is respectively $\mathrm{A}\left(\sigma_{1}, \tau_{1}\right)$ and $\mathrm{B}\left(\sigma_{2}, \tau_{2}\right)$, the equation for the simplified envelope is written as:

$$
\tau=\left[\frac{\tau_{2}-\tau_{1}}{\sigma_{2}-\sigma_{1}}\right] \sigma+\left[\tau_{1}-\sigma_{1} \frac{\tau_{2}-\tau_{1}}{\sigma_{2}-\sigma_{1}}\right]
$$

At early loading stages, the material is elastic, having a constant modulus of elasticity. Nonlinearity will occur when stress levels increase; the stiffness modulus will then decrease gradually. In the case of bi-axial tension or compression-tension, failure is initiated by cracking in the major tensile direction. In the minor principal direction the material remains un-cracked, resulting in an orthotropic condition. This state creates a differentiation of stiffness modulus in the direction of the principal axes. Orthotropic behavior is applied on the Möhr failure envelope model. On the model which employs Kupfer-Hilsdorf-Rusch failure envelope, the material is assumed isotropic subsequent to cracking, having an identical behavior in all directions. However, when exceeding the failure boundaries, the stiffness is adjusted based on the most dominant principal stress.

\section{Material Behavior}

\section{Material Nonlinearity}

Material nonlinearity is prominent for the compression stresses, since the tensile strength for concrete is relatively low. Nonlinearity is approached by the introduction of the nonlinearity index $\beta$, derived by Ottosen [3], relating the actual minor principal stress in compression to the compression stress at failure. At failure the value of $\beta=1$. The stiffness modulus of the material and the Poisson's ratio are derived as a function of the second invariant of the deviatoric stress tensor. The stress-strain relationship for the cementitious material is generated using the FIB Model Code 2010, while the stiffness modulus used in the orthotropic model is calculated from the stress-strain relation of the Tomaszewicz Model [4].

Tension behavior is approached by the fracture process, described using the stress-crack opening diagram. It was found that at stress levels of $90 \%$ to the tension strength, micro cracks significantly reduce the stiffness of the material [5]. The stress-strain relationship is therefore defined as a bilinear function. In the tension-compression zone the analysis was based on research work conducted by Vecchio and Collins [6].

\section{Isotropic and Orthotropic behavior}

If the bi-axial principal stresses at Gauss points enter the transition stage between the elastic and inelastic condition, the concrete can be assumed as an orthotropic material. A general formulation incorporating orthotropic behavior is as following [7].

$$
\frac{1}{1-v^{2}}\left[\begin{array}{ccc}
E_{1} & v \sqrt{E_{1} E_{2}} & 0 \\
v \sqrt{E_{1} E_{2}} & E_{2} & 0 \\
0 & 0 & \left(1-v^{2}\right)_{G}
\end{array}\right]
$$


$E_{1}$ and $E_{2}$ are the tangent modulus in the orthotropic axes, and $G$ is the stiffness modulus in shear. This approach will enable accommodation of the material behavior independently, depending on the stress condition in each principal direction. After cracking, the $E_{l}$ value is set to a substantially small number, representing failure in the major principal stress direction. This general formulation will also hold for the isotropic condition whereas $E_{1}=E_{2}=E$.

\section{Analysis and Discussion}

\section{Kupfer-Isotropic versus Möhr-Orthotropic approach}

The two developed finite element models were run for an identical case. The experimental research of an under - reinforced beam (code 4B2-05) conducted by Jang et. al. [8] was used as validation tool. Fig. 1. shows the load-displacement curves generated by the Kupfer-Isotropic and the Möhr-Orthotropic approach.

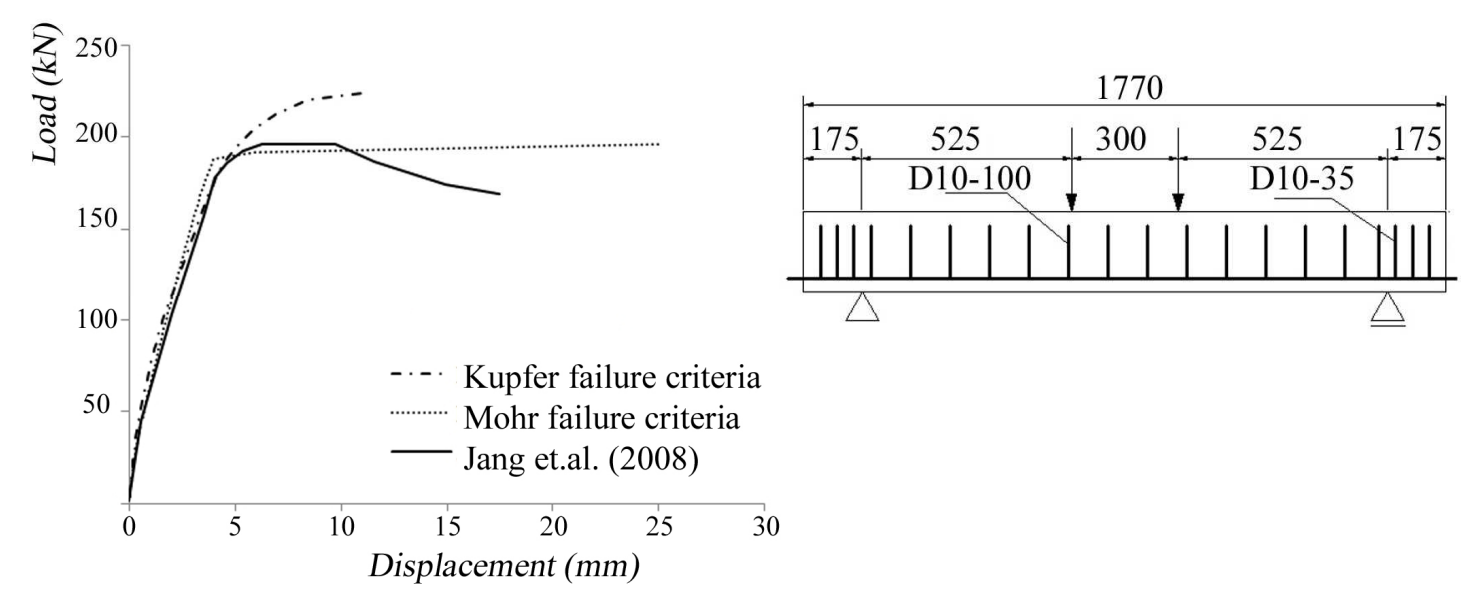

Fig. 1. Load-Displacement Curves Generated by the FE Model.

The simply supported beam has a concrete - cylinder strength of $40 \mathrm{MPa}$, and a length of $1770 \mathrm{~mm}$. The supports are a distance $1425 \mathrm{~mm}$ apart. The beam has a $140 \times 254.5 \mathrm{~mm}$ section, with 2D19 reinforcements, providing a steel - concrete ratio of 1.952. The steel has a yield and ultimate strength of respectively $435 \mathrm{MPa}$ and $618 \mathrm{MPa}$. The beam is loaded with the two-point loading system, distanced at $150 \mathrm{~mm}$ from the centre.

The program is developed to visually demonstrate the cracking pattern of the specimen under consideration. Fig. 2 shows the cracking pattern of the experimentally tested specimen, while Fig. 3 presents the cracking configuration predicted by the two models. The both generated models resulted in the same cracking formation, starting at the most extreme fibers in tension, expanding towards the neutral axes of the beam. At final stages, crushing of concrete in the compression zone was detected.

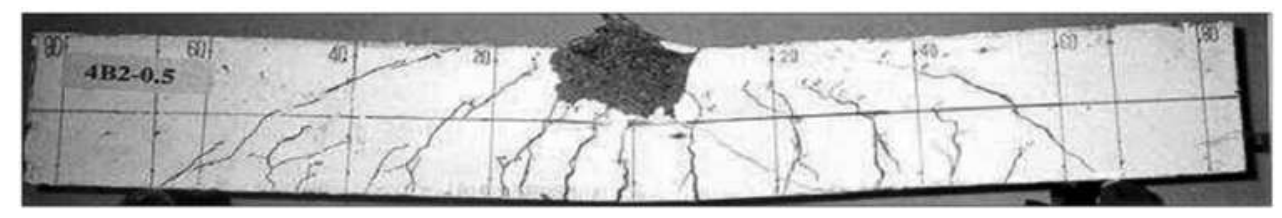

Fig. 2. Experimental Specimen (Jang et. al. 2008).

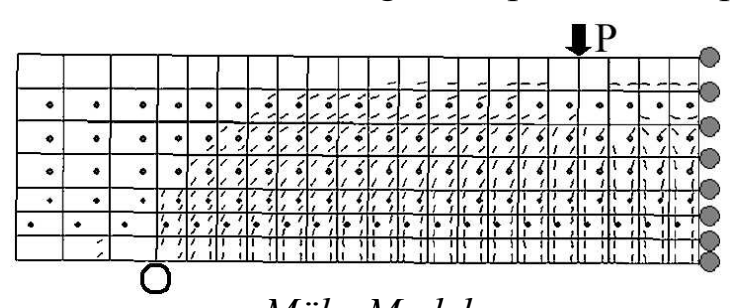

Möhr Model

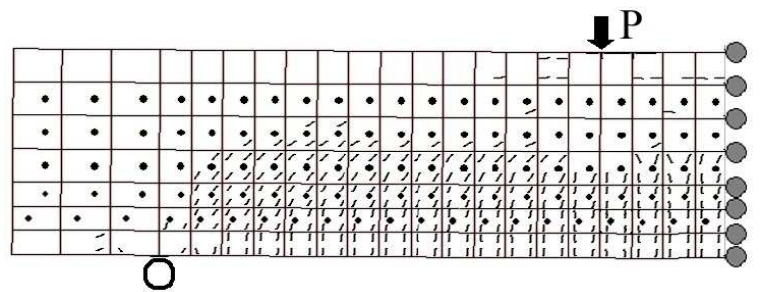

Kupfer Model

Fig. 3. Möhr versus Kupfer Cracking Pattern. 


\section{Load - Displacement Response}

The two load - displacement curves show a remarkably close representation to the actual behavior. The nonlinear response of the beam is reflected well, by both models. At early loading stages, up till $15 \%$ of the ultimate load, the curves demonstrate an identical behavior, than a small deviation is shown by the Kupfer-curve, exhibiting a higher structural stiffness when compared to the Möhr-curve. While the Möhr-curve has a distinctive yielding pattern followed by an almost flat line, the Kupfer-curve has a very smooth declining stiffness. The ultimate load was slightly conservative for the Möhr model, while the Kupfer model over predicted the ultimate load for about $12 \%$.

\section{Cracking Pattern}

The cracking configuration is well represented by both models. The experimental specimen shows compression damage at the upper most compressive fiber, and flexure cracks at the bottom fibers, extending beyond the neutral axes of the beam. Examining these failure patterns, it is suggested that for the same loading levels, the failure due to compression is more pronouncedly expressed by the Kupfer model, while flexure cracks are less severe. The Möhr model exhibits a more progressive flexure expansion, thus producing a better presentation of the flexural behavior under loading.

\section{Conclusion}

The approach used in the Möhr-Orthotropic model accommodates the adjustment of the material stiffness modulus in accordance to its individual stress behavior. The decrease in material stiffness is thus adjusted to the individual principal stresses. Also, this model takes into account the shear behavior at Gauss points.

When steel reinforcements reach their yield stress, large deformations in the surrounding areas of the bars will lead to rapid stress increase. If stresses exceed the Möhr envelope boundaries, the material stiffness modulus will come close to zero, further reducing the structural stiffness matrix significantly. The result is a very distinctive yield point, followed by an almost linear load displacement response. The model also predicts the ultimate load precisely, but overestimates the ultimate displacement of the specimen. The finite element model expresses the failure criteria by setting a substantially small value for the material stiffness modulus, technically preventing the program to accurately predict the final failure of the beam.

The cracking pattern resembles the real behavior closely. However, inconsistency in the compression areas is demonstrated. It is also noticed that crushing occurs at Gauss points that are not in the region of extreme compression fibers. The reason could be originated from the fact that the $M \ddot{h} r$ envelope does not directly accommodate the confinement effect provided by the principal stresses in compression.

Kupfer-Isotropic method failed to demonstrate the yielding of reinforcement. This is due to the fact that the isotropic approach only accommodates one type of material stiffness modulus, for a specific Gauss point. The model is built to adjust this stiffness modulus based on the most conservative possibility within the failure envelope. This assumption provides a virtual additional stiffness to the structural stiffness matrix, and creates a very gradually declining structural stiffness. The overestimation of ultimate load is most likely created by the confinement effect originated from the criterion set by the Kupfer envelope. However, the crack pattern in the compression zone of the beam is predicted more accurately by this model.

The both models are proven to be a sophisticated tool to predict the load - displacement curves and cracking pattern for under - reinforced concrete beams in flexure. The orthotropic material matrix suggests a more realistic approach to the real material behavior in bi-axial stresses. However, the $M \ddot{o} h r$ failure criteria deficient to accurately take into account the confinement effect granted by the compression stresses. 


\section{Future Research Work}

Studying the load - displacement curves and cracking pattern resulted by the two models, attempts should be made to develop a finite element model combining the Kupfer criteria, with the orthotropic material analysis, since such a model will have the benefits of the advantages of both systems. Also, validations of over - reinforced concrete beams should be included in the future study, to ensure the usability of the model for this type of specimens.

An alternate method to the smeared crack analysis, where the failed nodes of the element are removed from the system, should be explored. A node can be removed from the system when all surrounding element within the finite element meshing, have lost their stiffness. Thus instead of assigning a significantly small value for a failed Gauss point, a zero value is used. The removal of a node will reduce the structural stiffness matrix by 2 by 2 . Care has to be taken, since the removal of nodes can lead to a structural instability. To overcome this issue, a mesh generator can be used, to create smaller elements in the areas subjected to early failure, and to avoid poor elements.

A sensitivity analysis should be run, to provide information on the behavior of the model under variations of load increment and element size. The model should also be tested for specimens subjected to normal and flexural loading.

\section{Literature References}

[1] H. Kupfer, H.K. Hilsdorf, and H. Rusch: Behavior of Concrete under Biaxial Stresses, ACI Journal, Proceedings, Vol. 66, No. 8, (1969), p. 656.

[2] H. Fossen: Structural Geology, Cambridge University Press, (2010)

[3] N.S. Ottosen: Constitutive Model for Short-Time Loading of Concrete, Journal of the Engineering Mechanics Division, ASCE, Vol. 105, No. EM1, (1979), p. 127.

[4] K.K.B. Dahl: Uniaxial Stress-Strain Curves for Normal and High Strength Concretes, Rapport 7.6, Project 7, Department of Structural Engineering, Technical University of Denmark, (1992).

[5] A. Hillerborg, M. Modéer and P.E. Petersson: Analysis of crack formation and crack growth in concrete by means of fracture mechanics and finite elements, Cement and Concrete Research, Vol. 6, No. 6, (1976), p. 773.

[6] F.J. Vecchio and M.P. Collins: The Modified Compression-Field Theory for Reinforced Concrete Elements Subjected to Shear, ACI Journal Proceedings, Vol. 83, No. 2, (1986), p. 219.

[7] H.G. Kwak and F.C. Filippou: Finite Element Analysis of Reinforced Concrete Structures Under Monotonic and Cyclic Loads, $10^{\text {th }}$ World Conference in Earthquake Engineering, Madrid, Spain, (1992).

[8] I.Y. Jang, H.G. Park, S.S. Kim, J.H. Kim and Y.G. Kim: On the Ductility of High-Strength Concrete Beams. International Journal of Concrete Structure and Material, Vol. 2 No. 2. (2008), p. 115. 